\title{
Neural Basis of Ambivalence towards Ideal Self-Image in Schizophrenia
}

\author{
Byung-Hoon Kim¹, Yu-Bin Shin'1, Sunghyon Kyeong ${ }^{1}$, Seon-Koo Lee1, and Jae-Jin Kim ${ }^{1,2} \bowtie$ \\ ${ }^{1}$ Institute of Behavioral Science in Medicine, Yonsei University College of Medicine, Seoul, Republic of Korea \\ ${ }^{2}$ Department of Psychiatry, Yonsei University College of Medicine, Seoul, Republic of Korea
}

Objective Little has been explored about a reflection towards self-image in schizophrenia, though it can be related to heterogeneous symptoms of the illness. We identified the neural basis of ambivalence towards ideal self-image in patients with schizophrenia.

Methods 20 patients with schizophrenia and 20 healthy controls underwent functional MRI while the self-image reflection tasks of determining whether to agree with sentences describing their actual or ideal self-image that contained one of the adjective pairs with opposite valence. The interaction between the group and ideal ambivalence score was examined, and group differences in functional connectivity related to ambivalence towards ideal self-image were further studied.

Results The interaction of group-by-ideal ambivalence score was shown in the dorsal anterior cingulate cortex and dorsolateral prefrontal cortex, where activities were positively correlated with the level of ideal self-image ambivalence in patients, but not in controls. Task-related decrease in functional connectivity was shown between the orbitofrontal cortex and cerebellum in patients.

Conclusion The process of reflecting on ambivalent ideal self-image in schizophrenia may be related to aberrant prefrontal activity and connectivity. Abnormality in the prefrontal regions that take part in cognitive conflict monitoring and value judgment may underlie the pathophysiology of increased ambivalence towards ideal self-image.

Psychiatry Investig 2020;17(5):452-459

Key Words Schizophrenia, Self-image, Ambivalence, Functional magnetic resonance imaging.

\section{INTRODUCTION}

Schizophrenia is a psychiatric illness characterized by heterogeneous symptoms, including hallucinations, delusions, disorganized speech and behaviors, and negative symptoms. Although some advances have been made in understanding the disorder, a fundamental feature that can explain the root of the illness still remains enigmatic. One of the suspected core features underpinning the heterogeneous symptoms is selfdisturbance. ${ }^{1}$ While this psychopathology in schizophrenia has been explored in various perspectives including self-experience, self-awareness, and the sense of self, ${ }^{2}$ little is known about a reflection of self-image.

A reflection of self-image can be related to perceived emo-

Received: December 1, 2019 Revised: February 19, 2020

Accepted: March 8, 2020

$\triangle$ Correspondence: Jae-Jin Kim, MD, PhD

Department of Psychiatry, Yonsei University College of Medicine, Gangnam Severance Hospital, 211 Eonju-ro, Gangnam-gu, Seoul 06273, Republic of Korea

Tel: +82-2-2019-3341, Fax: +82-2-3462-4304, E-mail: jaejkim@yonsei.ac.kr

(a) This is an Open Access article distributed under the terms of the Creative Commons Attribution Non-Commercial License (https://creativecommons.org/licenses/bync/4.0) which permits unrestricted non-commercial use, distribution, and reproduction in any medium, provided the original work is properly cited. tion, social anxiety, feelings of guilt, or self-esteem. ${ }^{3}$ In particular, the self-discrepancy theory postulates the domains of the self as the actual-, ideal-, and ought-self, which refer to the representations of self-image that one actually possesses, would like to possess, and ought to possess, respectively. ${ }^{4}$ The discrepancy between the actual-self and ideal-self has been related to psychiatric symptoms such as paranoia and depression. ${ }^{5-7}$ From this viewpoint, we can expect that a reflection of actual or ideal self-image may also be disturbed in schizophrenia. However, little has been studied regarding a disturbance of self-image reflection in the illness. To address this issue, the present study focused on evaluating the level of ambivalence that patients with schizophrenia show towards one's self-image.

Ambivalence, which is a state of having opposite feelings or beliefs at the same time towards some object or person, has long been considered to be an important characteristic in schizophrenia. ${ }^{8}$ Previous studies have shown that the increased level of ambivalence in schizophrenia is related to various symptoms such as schizotypy, anhedonia, and negative mood., ${ }^{910}$ Measuring the level of ambivalence in most of the previous studies has been done with the Schizotypal Ambivalence Scale (SAS), ${ }^{11}$ which evaluates ambivalence from a scope of schizo- 
typy rather than ambivalence towards one's self-image reflection concerned in the present study. In fact, it can be hardly said that there exists a validated measure of ambivalence for use in patients with schizophrenia. ${ }^{8,9}$ Furthermore, no functional neuroimaging experiment to find a brain region relevant to ambivalence towards self-image has been carried out to date.

The impairment in the self-referential processing and/or value judgment can lead to contradictive value assignment to one's ideal self-image, giving rise to the ambivalent feeling towards ideal self-image. ${ }^{12}$ This inherent contradictive value assignment can possibly be accompanied by recruiting the cognitive conflict monitoring function to resolve the contradiction. ${ }^{13}$ Therefore, it can be expected that the neural substrates of ambivalence towards ideal self-image in schizophrenia would be found in brain regions related to three cognitive processes, which are self-referential processing, value judgment, and cognitive conflict monitoring. In fact, there have been reports of impaired self-referential processing in schizophrenia based on aberrant activity in the midline cortical structures, including the medial prefrontal cortex (MPFC) and posterior cingulate cortex (PCC) ${ }^{14,15}$ It has also been reported that patients with schizophrenia show impaired value judgment based on orbitofrontal cortex (OFC) dysfunction ${ }^{16,17}$ and abnormal cognitive conflict monitoring associated with dorsal anterior cingulate cortex (ACC) and dorsolateral prefrontal cortex (DLPFC) dysfunctions. ${ }^{18-21}$

The present study aimed to objectively measure the level of ambivalence towards positive or negative self-image with a behavioral task and explore the neural mechanism of ideal selfimage reflection in schizophrenia. For this approach, we used functional magnetic resonance imaging (fMRI) with a self-image reflection task, in which the attitudes towards actual or ideal self-image were evaluated. We hypothesized that patients with schizophrenia would exhibit higher levels of ambivalence towards actual and ideal self-image than healthy controls. It was also hypothesized that the level of ambivalence towards ideal self-image would exhibit a different pattern in patients with schizophrenia compared to controls regarding regional activity and functional connectivity related to self-referential processing (i.e., MPFC and PCC), value judgment (i.e., OFC), and cognitive conflict monitoring (i.e., dorsal ACC and DLPFC).

\section{METHODS}

\section{Participants}

Participants included 20 patients with schizophrenia and 20 age- and sex-matched healthy controls. Patients were recruited at psychiatric outpatient clinics after a psychiatric interview confirming the diagnosis of schizophrenia. All partic- ipants underwent the Structured Clinical Interview for DSM$I^{22}$ to exclude psychiatric diagnoses other than schizophrenia. Exclusion criteria included current or past substance abuse or dependence, presence of significant neurological or medical illnesses, and any contraindication for MRI scanning. This study was approved by the Institutional Review Board of Severance Hospital, Yonsei University and informed consent was obtained from all participants (4-2013-0875).

\section{Psychometric self-report scales and clinician-rated symptom severity}

All participants completed self-reporting psychometric scales, including the SAS ${ }^{11}$ and the Beck Depression Inventory $(B D I)^{23}$ to measure the severity of subjective ambivalent feelings and depressive symptoms, respectively. Differences in the scores between the two groups were compared with independent t-tests (Table 1). Patients were further evaluated for symptom severity with the Positive and Negative Syndrome Scale $(\mathrm{PANSS})^{24}$ and Global Assessment of Functioning (GAF). ${ }^{25}$

\section{Task design and behavioral data analysis}

Participants underwent a set of self-image reflection tasks during fMRI scanning of the brain. The task stimuli were a total of 96 sentences; each sentence included an adjective that described actual or ideal self-image of the participant. The adjectives comprised 24 word pairs of opposite valence (e.g., optimistic-pessimistic), and sentences consisted of four types, such as actual positive (e.g., "I am an optimistic person"), actual negative (e.g." "I am a pessimistic person"), ideal positive (e.g., "I wish to be an optimistic person"), and ideal negative (e.g., "I wish to be a pessimistic person"). The task stimuli were presented as a block design with two sessions. The block condition was set for each stimulus type. Each block consisted of six sentences, each of which was displayed for four seconds. A total of eight experimental blocks in each session were randomly presented. Resting blocks using a cross-hair were presented for 16 seconds between task blocks. In response to the sentences, participants were asked to choose one of the four answers: 1) strongly disagree; 2) disagree; 3) agree; and 4) strongly agree.

The behavioral performance was analyzed in terms of the response score and reaction time. The response score was evaluated as follows: 'strongly disagree $=-2$,' 'disagree $=-1$, ' agree $=+1$,' and 'strongly agree $=+2$.' The actual and ideal ambivalence scores were elicited in each of the actual and ideal conditions, respectively, as the sum of 24 conversion values, which were a multiplication of the two response scores corresponding to the word pair of opposite valence. This formula was based on a cross-product model of the models for calculating ambivalence. ${ }^{26}$ Reaction time of each participant was also calculated 
Table 1. Demographic and clinical variables of participants

\begin{tabular}{|c|c|c|c|c|c|}
\hline & Schizophrenia $(\mathrm{F} / \mathrm{M}=10 / 10)$ & Healthy control $(\mathrm{F} / \mathrm{M}=10 / 10)$ & $\mathrm{t}$ & $\mathrm{df}$ & $\mathrm{p}$ \\
\hline Age (y) & $34.90 \pm 8.20$ & $31.95 \pm 7.23$ & 1.21 & 38 & 0.235 \\
\hline Education (y) & $12.50 \pm 2.33$ & $16.25 \pm 2.40$ & -5.01 & 38 & $<0.001$ \\
\hline SAS score & $10.50 \pm 4.84$ & $5.05 \pm 4.71$ & 3.61 & 38 & $<0.001$ \\
\hline BDI score & $19.20 \pm 8.64$ & $7.25 \pm 5.41$ & 5.24 & 38 & $<0.001$ \\
\hline PANSS score & $79.60 \pm 9.51$ & - & - & - & - \\
\hline GAF score & $45.80 \pm 8.23$ & - & - & - & - \\
\hline Actual AS & $-8.00 \pm 17.26$ & $-28.75 \pm 17.01$ & 3.83 & 38 & $<0.001$ \\
\hline Ideal AS & $-26.25 \pm 23.274$ & $-57.45 \pm 17.68$ & 4.77 & 38 & $<0.001$ \\
\hline Actual RT (ms) & $1,868.04 \pm 464.83$ & $1,513.70 \pm 259.97$ & 2.98 & 38 & 0.005 \\
\hline Ideal RT (ms) & $1,790.44 \pm 438.31$ & $1,411.85 \pm 218.96$ & 3.46 & 38 & 0.002 \\
\hline
\end{tabular}

F: female, M: male, SAS: Schizotypal Ambivalence Scale, BDI: Beck Depression Inventory, PANSS: Positive and Negative Syndrome Scale, GAF: Global Assessment of Functioning, AS: ambivalence score, RT: reaction time

separately in the two conditions. Independent t-tests between the schizophrenia and control groups were performed to compare the mean values of the actual and ideal ambivalence scores and reaction times in the two conditions. Pearson correlation analysis was performed to clarify the relationship of the actual and ideal ambivalence scores and reaction times with psychometric scale scores (SAS, BDI, PANSS, and GAF).

\section{Image acquisition and preprocessing}

Brain images were obtained using a 3.0 T MRI scanner (Intera Achieva; Philips Medical System, Best, Netherlands). Whole-brain functional imaging data were acquired with an echo planar pulse sequence (echo time $=30 \mathrm{~ms}$, repetition time $=2,000 \mathrm{~ms}$, field of view $=240 \mathrm{~mm}$, matrix $=64 \times 64$, number of slices $=30$, slice thickness $=4 \mathrm{~mm}$ ). High resolution anatomical images were acquired with a T1-weighted gradientecho sequence (echo time $=4.6 \mathrm{~ms}$, repetition time $=9.7 \mathrm{~ms}$, flip angle $=80^{\circ}$, field of view $=220 \mathrm{~mm}$, matrix $=224 \times 224$, slice thickness $=1.20 \mathrm{~mm}$ ) to serve as an anatomical underlay for the fMRI data.

Spatial preprocessing and statistical analysis of functional images were performed with SPM12 (Wellcome Trust Centre for Neuroimaging; http://www.fil.ion.ucl.ac.uk/spm). After discarding the first five volumes from the dummy scan at each session, the remaining 152 volumes were used for further preprocessing. After realigning on the first image and slice-timing correction, functional images were co-registered to structural images for each subject. The spatial normalization of coregistered images was done with nonlinear transformation functions obtained by registering the individual T1-weighted images to a standard Montreal Neurological Institute (MNI) template. These normalized data were smoothed with a $6 \mathrm{~mm}$ full-width at half-maximum kernel.

\section{Imaging results and statistical analysis}

Preprocessed images were analyzed using a general linear model. A series of events as regressors of interest was modeled by convolving the event-train of stimulus onsets with the canonical hemodynamic response function separately for each session. Trials were modeled based on the onset of stimulus presentation, specified as zero-duration events, with movement parameters as regressors of no-interest. The first-level analysis generated within-subject contrast maps of whole-brain activity during ideal self-image reflection, with activity during actual self-image reflection serving as the control condition.

The second-level analysis was conducted for the wholebrain as the interaction effect between the group and ideal ambivalence score. The BDI score was used as a control variable in this analysis, considering that the degree of depression can affect the ambivalence score. Results were considered significant at a threshold of family-wise error-corrected $\mathrm{p}<0.05$. Then, the parameter estimate of activity in the statistically significant clusters was determined for each subject for further analysis. Post-hoc correlation analysis between the regional activity and the ideal ambivalence score was performed in the separate groups. In addition, we performed correlation analysis between the regional activity and the SAS score.

The group difference of functional connectivity with respect to the level of ambivalence towards ideal self-image was further studied using the CONN toolbox. ${ }^{27}$ The intrinsic connectivity analysis with the advantage of not requiring prior information about the seed region of functional connectivity ${ }^{28,29}$ was first performed to find the seed region. In this analysis, intrinsic connectivity of a voxel was defined as the root mean square of the correlation coefficients of the voxel with all other voxels in the brain, representing a measure of node centrality of the voxel..$^{28}$ In this analysis, voxels showing a significant group difference for the regression of the ideal ambivalence 
score with respect to intrinsic connectivity were specified as the seed region. Then, the group difference in seed-based functional connectivity during the ideal task condition was identified using independent t-test.

\section{RESULTS}

\section{Demographic and clinical variables}

Participants with schizophrenia did not significantly differ from healthy controls in terms of age or sex ratio, while educational level was significantly higher in the control group $(\mathrm{t}=-5.01, \mathrm{p}<0.001)$. Independent $\mathrm{t}$-tests of self-reported scales showed significant differences indicating higher levels of schizotypal ambivalence $(\mathrm{t}=3.61, \mathrm{p}<0.001)$ and depression $(\mathrm{t}=5.24, \mathrm{p}<0.001)$ in the schizophrenia group (Table 1$)$.

\section{Behavioral task results}

The schizophrenia group had significantly higher scores for actual ambivalence $(\mathrm{t}=3.83, \mathrm{p}<0.001)$ and ideal ambivalence $(\mathrm{t}=4.77, \mathrm{p}<0.001)$ than the control group (Figure 1). Reaction time was significantly longer in both actual $(\mathrm{t}=2.98, \mathrm{p}=0.005)$ and ideal $(\mathrm{t}=3.46, \mathrm{p}=0.002)$ conditions in the schizophrenia group than in the control group.

In the correlation analysis, the actual ambivalence score was positively correlated with the SAS score $(r=0.45, p=0.045)$ in the control group and negatively correlated with the GAF score $(\mathrm{r}=-0.48, \mathrm{p}=0.031)$ in the schizophrenia group, whereas the ideal ambivalence score was not correlated with any clinical scores in either group. In the correlation between reaction time and clinical score, the control group showed a negative correlation between reaction time for the actual condition and the SAS score ( $r=-0.46, \mathrm{p}=0.044)$, whereas the schizophrenia group showed positive correlations between reaction time of both actual $(\mathrm{r}=0.54, \mathrm{p}=0.014)$ and ideal $(\mathrm{r}=0.68, \mathrm{p}=0.004)$ conditions and the BDI score. Other correlations were not significant in either group.

\section{Regional activity during ambivalence}

The dorsal ACC and bilateral DLPFC showed a significant interaction effect between group and ideal ambivalence score (Table 2). In the post-hoc partial correlation analysis for each group, the schizophrenia group showed a significant positive correlation of functional activity with the ideal ambivalence score in all regions, such as the dorsal ACC ( $\mathrm{r}=0.71, \mathrm{p}=0.001)$, left DLPFC $(r=0.65, p=0.003)$, and right DLPFC $(r=0.68, p=$

Table 2. Brain regions exhibiting the significant interaction effect of correlation with the ideal ambivalence score

\begin{tabular}{llcccccc}
\hline \multirow{2}{*}{ Region } & \multirow{2}{*}{$\begin{array}{c}\text { Side } \\
\text { Cluster }\end{array}$} & $\mathrm{Z}_{\max }$ & \multicolumn{4}{c}{ Coordinates } \\
\cline { 5 - 8 } & & size & & $\mathrm{x}$ & $\mathrm{y}$ & $\mathrm{z}$ \\
\hline $\begin{array}{l}\text { Dorsal anterior cingulate } \\
\text { cortex }\end{array}$ & Right & 884 & 4.88 & 4 & 20 & 40 \\
$\begin{array}{l}\text { Dorsolateral prefrontal } \\
\text { cortex }\end{array}$ & Left & 447 & 4.49 & -40 & 38 & 8 \\
& Right & 487 & 4.70 & 36 & 32 & 22 \\
\hline
\end{tabular}

Cluster size is the number of voxels. $\mathrm{Z}_{\max }$ and Coordinates mean Z-value and Montreal Neurological Institute (MNI) coordinates (x, $\mathrm{y}, \mathrm{z}$ in $\mathrm{mm}$ ) of the peak voxel in the cluster, respectively. Statistical inferences were thresholded using a significance of family-wise error (FWE) corrected $\mathrm{p}<0.05$
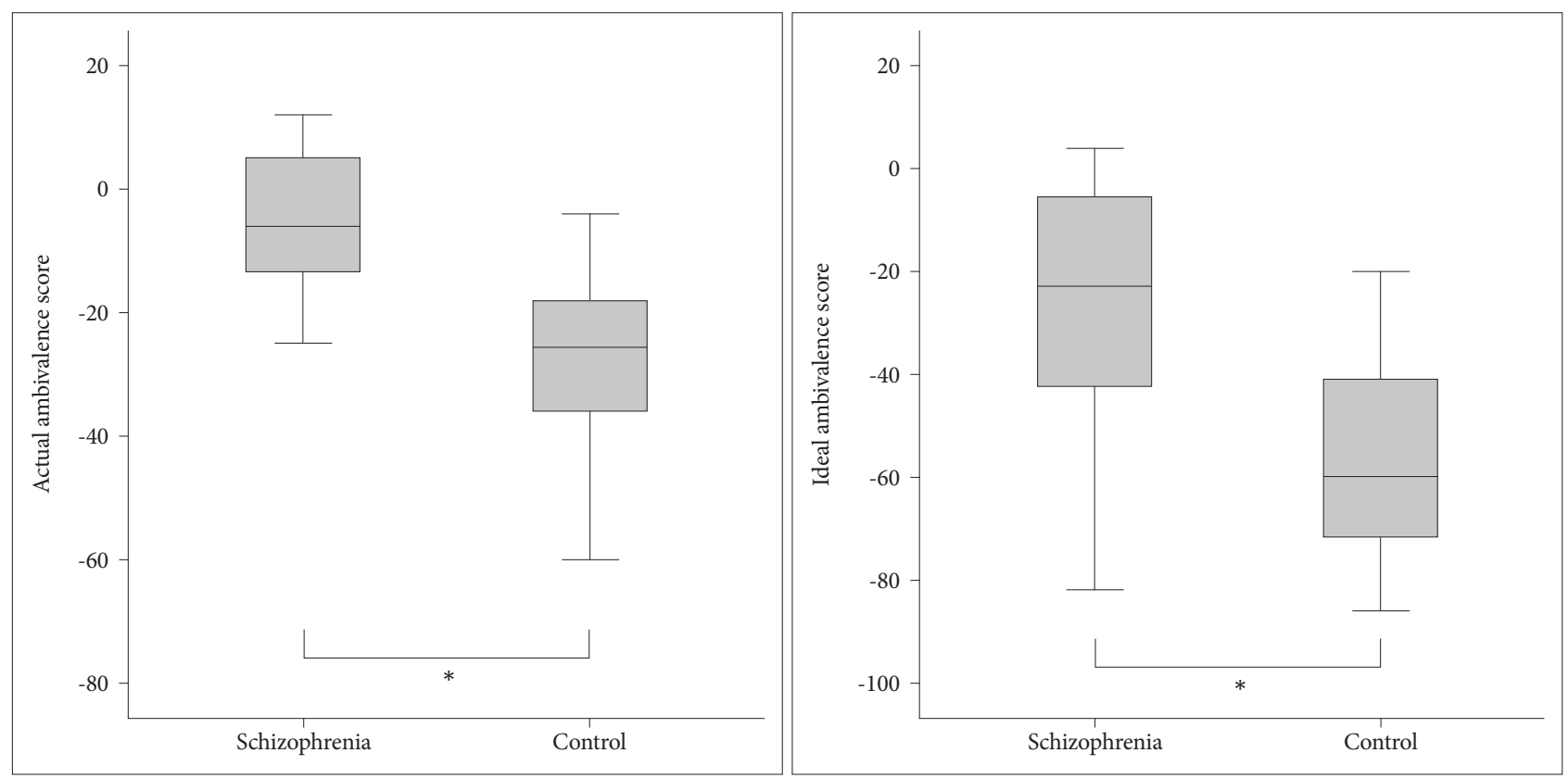

Figure 1. Behavioral results for the self-image reflection task. Both actual and ideal ambivalence scores were significantly higher in the schizophrenia group than in the control group. ${ }^{*} p<0.05$. 
0.001), whereas the control group showed no significant correlations (Figure 2A). Meanwhile, in the partial correlation between regional activity and the SAS score, the schizophrenia group showed a significant negative correlation in the left $\operatorname{DLPFC}(\mathrm{r}=-0.50, \mathrm{p}=0.028)$ and right DLPFC $(\mathrm{r}=-0.48, \mathrm{p}=0.040)$, but not in the dorsal ACC. The control group showed no significant correlation.

\section{Functional connectivity during ambivalence}

The intrinsic connectivity analysis showed a significant group difference in functional connectivity in the left OFC area. Then, the functional connectivity analysis using the OFC area as the seed region revealed reduced connectivity with the right cerebellum in the schizophrenia group when compared with the control group (Figure 2B).

\section{DISCUSSION}

In the present study for exploring a measured level of ambivalence towards self-image reflection and its neural basis in schizophrenia, utilization of the ambivalence score, which was derived from the behavioral data, was a unique approach for quantifying the objective level of ambivalence. This study ob- jectively demonstrated the level of ambivalence towards actual and ideal self-image in patients with schizophrenia and healthy controls. As hypothesized, patients showed a significantly higher level of ambivalence towards both actual self-image and ideal self-image in the behavioral task than controls. This result is consistent with previous studies using the other measurements that have indicated elevated levels of ambivalence in patients with schizophrenia. ${ }^{8,930}$ Although caution is warranted since the uniqueness of our approach may require further verification to ensure it accurately represents an individual's level of ambivalence, we believe that this kind of new attempt is needed to advance the study for understanding ambivalence.

The interaction effect between group and ideal ambivalence score was found only in the dorsal ACC and bilateral DLPFC, where regional activity and the ideal ambivalence score were positively correlated in the schizophrenia group, but not in the control group. These two regions have been known to mediate conflict processing on cognitive control. ${ }^{13,31}$ A previous study using the synaptic specializations also suggest that the two regions have the unique roles in mechanisms of cognitive control. ${ }^{32}$ In particular, the dorsal ACC is engaged in executive control occurring at response-related levels of processing, ${ }^{33}$ and is a critical region of the distributed conscious control

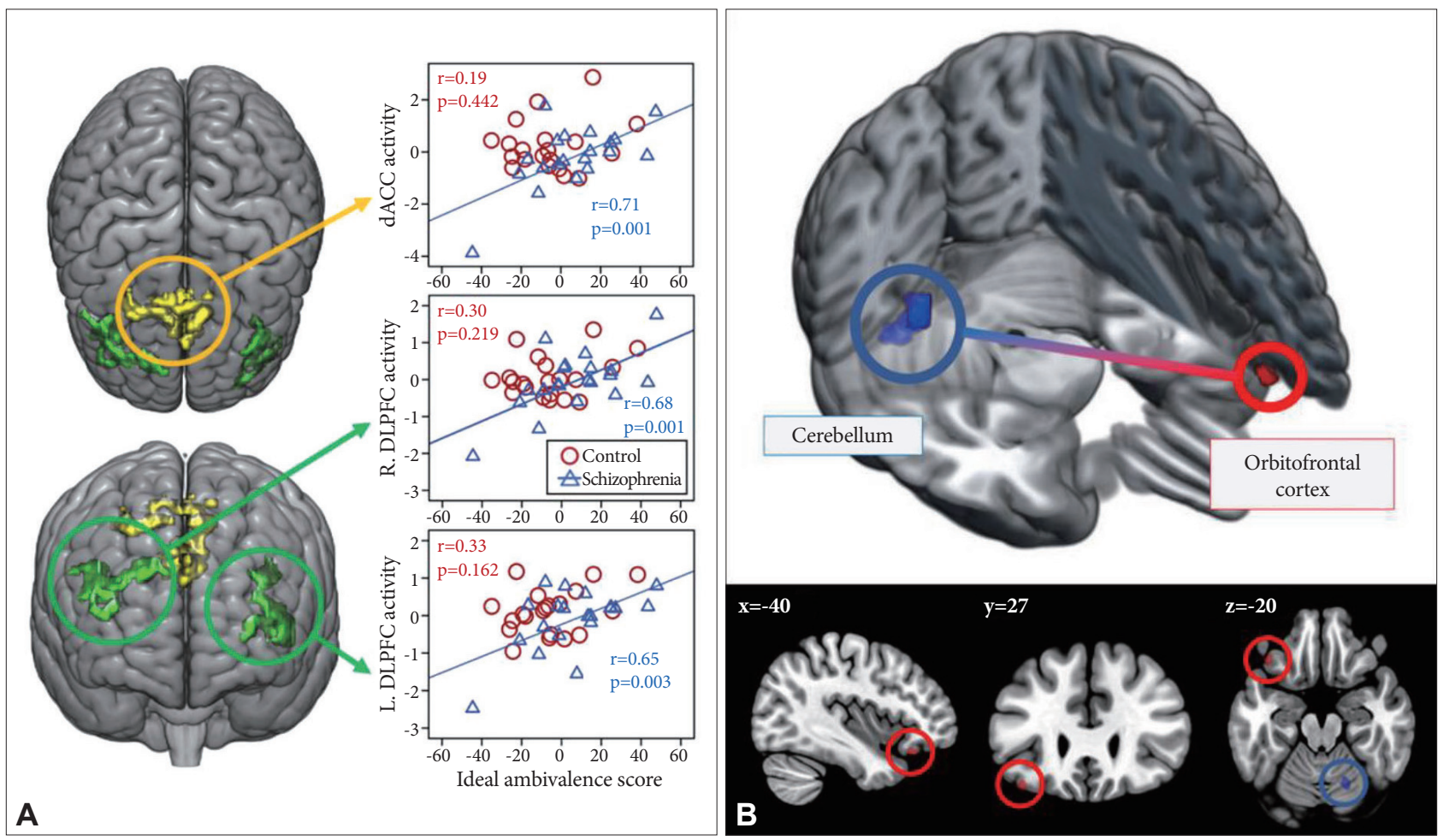

Figure 2. Summary of imaging results. A: Ideal ambivalence score and its partial correlation with activity of the dorsal anterior cingulate cortex (dACC) and left (L.) and right (R.) dorsolateral prefrontal cortex (DLPFC) in each group. The schizophrenia group showed a significant positive correlation of functional activity with the ideal ambivalence score in all regions (blue triangles), whereas the control group showed no significant correlations (red circle). B: Results from the intrinsic connectivity analysis and functional connectivity analysis. Significant voxels in the intrinsic connectivity analysis are shown in red (the left orbitofrontal cortex), whereas those in the functional connectivity analysis are shown in blue (the right cerebellum). 
network that is disrupted in schizophrenia. ${ }^{34}$ The DLPFC is involved in executive control including hierarchically ordered control signals, ${ }^{35}$ and executive dysfunctions such as impairments in contextual control ${ }^{36}$ or proactive control ${ }^{37}$ have been considered to be a common feature of schizophrenia. Therefore, our results from the analysis of regional activity suggest that ambivalence towards ideal self-image in patients with schizophrenia may be associated with dysfunctions in regions related to cognitive conflict monitoring or executive control rather than self-referential processing and value judgment. Given that altered ACC and PFC activity during cognitive control may be a potential indicator of genetic liability for schizophrenia, ${ }^{21}$ ambivalence towards ideal self-image is also likely to have a genetic basis in patients with schizophrenia.

It is worth noting that the self-reported SAS score and DLPFC activity showed a negative correlation, not a positive correlation. Although both the ambivalence score and SAS score were applied to measure the level of one's ambivalence, the former was about ambivalence towards self-image in our behavioral task, whereas the latter dealt with ambivalence related to schizotypal traits. ${ }^{38}$ Our behavioral results showed that the two scores were not correlated in the schizophrenia group. The SAS includes some items quantifying ambiguity of one's feelings or thoughts rather than ambivalence (e.g., I doubt if I can ever be sure exactly what my true interests are). Therefore, the two scores seem to measure completely different aspects of ambivalence that may be different in direction. In this aspect, the negative correlation between the SAS score and DLPFC activity may support that the more severe the schizotypal ambivalence traits, the weaker the execution-related DLPFC activity.

In contrast to our expectations, the medial cortical structures such as the MPFC and PCC that play a role in self-referential processing ${ }^{39,40}$ showed no interaction effect between group and ideal ambivalence score. This result may be because that the element of self-referential processing was eliminated in analyzing the imaging data. Brain activity during actual selfimage reflection was used as the control in the first-level analysis generating within-subject contrast maps for ideal self-image reflection. If the other task independent of self-image was used as a control condition, MPFC or PCC activity might have been the result.

Although the hypothesis that dysfunction of the brain region related to value judgment would be related to ambivalence towards ideal self-image was not proved in the results of regional activity, it was not in the results of functional connectivity. The left OFC, which is related to the value judgment function, ${ }^{41}$ was found to have aberrant intrinsic connectivity in the schizophrenia group. Furthermore, the functional connectivity analysis based on the left OFC showed decreased connectivity with the cerebellum in the schizophrenia group.
The dysfunction of the OFC and cerebellum with respect to ambivalence has also been revealed in the results of our previous research. ${ }^{30}$ Given that cerebellar dysfunction in schizophrenia may cause coordination failure of mental or cognitive processes, ${ }^{42,43}$ decreased functional connectivity between the OFC and cerebellum may represent the disruption of cognitive coordination related to value judgment. This disruption can in turn be expressed as elevated level of ambivalence towards ideal self-image in schizophrenia.

Although our neuroimaging experiment was designed to identify the brain regions related to the level of ambivalence towards ideal self-image, the regions revealed from the analyses are all known to be related to anhedonia, a representative negative symptom of schizophrenia. ${ }^{44}$ In terms of anhedonia, the OFC is responsible for judging the value of a stimuli ${ }^{45-47}$ and sending the coded value information to the DLPFC and the ACC, where reward valuation is processed for the final decision-making. ${ }^{44}$ The cerebellum is also gaining much attention recently as a central coordinating region of the negative symptom in schizophrenia. ${ }^{48}$ It has been raised that the status of anhedonia should be clarified in studying affective ambivalence in patients with schizophrenia. ${ }^{8}$ We carefully suggest it is worth noting from our study that there may exist a shared underlying neural pathophysiology between anhedonia and ambivalence.

There are some limitations to be discussed in this study. First, the sample size was relatively small, and thus caution is warranted when generalizing our findings. Second, the schizophrenia group had a lower educational level and higher depression score than the control group, and the level of cognitive functioning was not considered in the study. Finally, the medications that patients were taking were not included in the analysis. There is evidence that antipsychotic drugs can alter functional activity and connectivity of the brain in patients with schizophrenia, ${ }^{49}$ which could have confounded the results of our study.

In summary, our experiment demonstrated that patients with schizophrenia showed the positive correlation of activity in the dorsal ACC and DLPFC with the level of ambivalence towards ideal self-image and aberrant functional connectivity between the OFC and cerebellum. These results suggest that aberrant functional activity and connectivity in the brain regions that take part in cognitive conflict monitoring and value judgment underlie the pathophysiology of increased ambivalence towards ideal self-image in patients with schizophrenia.

\section{Acknowledgments}

This work was supported by the National Research Foundation of Korea (NRF) grant funded by the Korea government (MSIP) (No. NRF2016R1A2A2A10921744). 


\section{Conflicts of Interest}

The authors have no potential conflicts of interest to disclose.

\section{Author Contributions}

Conceptualization: Jae-Jin Kim. Data curation: Seon-Koo Lee. Formal analysis: Byung-Hoon Kim. Funding acquisition: Jae-Jin Kim. Investigation: Yu-Bin Shin. Methodology: Sunghyon Kyeong. Project administration: Seon-Koo Lee. Resources: Yu-Bin Shin. Software: Sunghyon Kyeong. Supervision: Jae-Jin Kim. Validation: Sunghyon Kyeong. Visualization: Byung-Hoon Kim. Writing—orininal draft: Byung-Hoon Kim. Writingreview \& editing: Jae-Jin Kim.

\section{ORCID iDs}

Byung-Hoon Kim https://orcid.org/0000-0003-2501-038X Yu-Bin Shin https://orcid.org/0000-0002-7311-9438

Sunghyon Kyeong https://orcid.org/0000-0002-9095-5219

Seon-Koo Lee https://orcid.org/0000-0002-1493-3464

Jae-Jin Kim https://orcid.org/0000-0002-1395-4562

\section{REFERENCES}

1. Sass LA, Parnas J. Schizophrenia, consciousness, and the self. Schizophr Bull 2003;29:427-444.

2. Moe AM, Docherty NM. Schizophrenia and the sense of self. Schizophr Bull 2014;40:161-168.

3. Tangney JP, Niedenthal PM, Covert MV, Barlow DH. Are shame and guilt related to distinct self-discrepancies? A test of Higgins's (1987) hypotheses. J Pers Soc Psychol 1998;75:256-268.

4. Higgins ET. Self-discrepancy: a theory relating self and affect. Psychol Rev 1987;94:319-340.

5. Bhar SS, Kyrios M. An investigation of self-ambivalence in obsessivecompulsive disorder. Behav Res Ther 2007;45:1845-1857.

6. Kinderman P, Prince S, Waller G, Peters E. Self-discrepancies, attentional bias and persecutory delusions. Br J Clin Psychol 2003;42:1-12.

7. Tiernan B, Tracey R, Shannon C. Paranoia and self-concepts in psychosis: a systematic review of the literature. Psychiatry Res 2014;216: 303-313.

8. Trémeau F, Antonius D, Cacioppo JT, Ziwich R, Jalbrzikowski M, Saccente E, et al. In support of Bleuler: objective evidence for increased affective ambivalence in schizophrenia based upon evocative testing. Schizophr Res 2009;107:223-231.

9. Docherty AR, Cicero DC, Becker TM, Kerns JG. Self-reported ambivalence in schizophrenia and associations with negative mood. J Nerv Ment Dis 2014;202:70-73.

10. Docherty AR, Sponheim SR, Kerns JG. Further examination of ambivalence in relation to the schizophrenia spectrum. Schizophr Res 2014;158:261-263.

11. Raulin ML, Brenner V. Ambivalence. In: Costello CG, Editor. Symptoms of Schizophrenia. New York: Wiley, 1993, p.201-226.

12. D'Argembeau A. On the role of the ventromedial prefrontal cortex in self-processing: the valuation hypothesis. Front Hum Neurosci 2013; $7: 372$.

13. Mansouri FA, Tanaka K, Buckley MJ. Conflict-induced behavioural adjustment: a clue to the executive functions of the prefrontal cortex. Nat Rev Neurosci 2009;10:141-152.

14. Holt DJ, Cassidy BS, Andrews-Hanna JR, Lee SM, Coombs G, Goff $\mathrm{DC}$, et al. An anterior-to-posterior shift in midline cortical activity in schizophrenia during self-reflection. Biol Psychiatry 2011;69:415-423.

15. Shad MU, Keshavan MS, Steinberg JL, Mihalakos P, Thomas BP, Motes MA, et al. Neurobiology of self-awareness in schizophrenia: an fMRI study. Schizophr Res 2012;138:113-119.

16. Albrecht MA, Waltz JA, Frank MJ, Gold JM. Probability and magnitude evaluation in schizophrenia. Schizophr Res Cogn 2016;5:41-46.

17. Strauss GP, Robinson BM, Waltz JA, Frank MJ, Kasanova Z, Herbener
ES, et al. Patients with schizophrenia demonstrate inconsistent preference judgments for affective and nonaffective stimuli. Schizophr Bull 2011;37:1295-1304.

18. Dehaene S, Artiges E, Naccache L, Martelli C, Viard A, Schürhoff F, et al. Conscious and subliminal conflicts in normal subjects and patients with schizophrenia: the role of the anterior cingulate. Proc Natl Acad Sci U S A 2003;100:13722-13727.

19. Kerns JG, Cohen JD, MacDonald AW 3rd, Johnson MK, Stenger VA, Aizenstein $\mathrm{H}$, et al. Decreased conflict- and error-related activity in the anterior cingulate cortex in subjects with schizophrenia. Am J Psychiatry 2005;162:1833-1839.

20. Miller EK. The prefrontal cortex and cognitive control. Nat Rev Neurosci 2000;1:59-65.

21. Sambataro F, Mattay VS, Thurin K, Safrin M, Rasetti R, Blasi G, et al. Altered cerebral response during cognitive control: a potential indicator of genetic liability for schizophrenia. Neuropsychopharmacology 2013;38:846-853.

22. Spitzer RL, Williams JBW, Gibbon M, First M. Structured Clinical Interview for DSM-IV. New York: Biometrics Research; 1994

23. Beck AT, Ward CH, Mendelson M, Mock J, Erbaugh J. An inventory for measuring depression. Arch Gen Psychiatry 1961;4:561-571.

24. Kay SR, Fiszbein A, Opler LA. The positive and negative syndrome scale (PANSS) for schizophrenia. Schizophr Bull 1987;13:261-276.

25. Spitzer RL, Gibbon M, Williams JBW, Endicott J. Global Assessment of Functioning (GAF) Scale. In: Sedere LI, Dickey B, Editors. Outcomes Assessment in Clinical Practice. Baltimore: Williams \& Wilkins, 1996, p.76-78.

26. Priester JR, Petty RE. The gradual threshold model of ambivalence: relating the positive and negative bases of attitudes to subjective ambivalence. J Pers Soc Psychol 1996;71:431-449.

27. Whitfield-Gabrieli S, Nieto-Castanon A. Conn: a functional connectivity toolbox for correlated and anticorrelated brain networks. Brain Connect 2012;2:125-141.

28. Martuzzi R, Ramani R, Qiu M, Shen X, Papademetris X, Constable RT. A whole-brain voxel based measure of intrinsic connectivity contrast reveals local changes in tissue connectivity with anesthetic without a priori assumptions on thresholds or regions of interest. Neuroimage 2011;58:1044-1050.

29. Marchand WR, Lee JN, Johnson S, Gale P, Thatcher J. Differences in functional connectivity in major depression versus bipolar II depression. J Affect Disord 2013;150:527-532.

30. Kim JJ, Park HJ, Jung YC, Chun JW, Kim HS, Seok JH, et al. Evaluative processing of ambivalent stimuli in patients with schizophrenia and depression: a [ $\left.{ }^{15} \mathrm{O}\right] \mathrm{H}_{2} \mathrm{O}$ PET study. J Int Neuropsychol Soc 2009;15: 990-1001.

31. Chun JW, Park HJ, Kim DJ, Kim E, Kim JJ. Contribution of fronto-striatal regions to emotional valence and repetition under cognitive conflict. Brain Res 2017;1666:48-57.

32. Medalla M, Barbas H. Synapses with inhibitory neurons differentiate anterior cingulate from dorsolateral prefrontal pathways associated with cognitive control. Neuron 2009;61:609-620.

33. van Veen V, Cohen JD, Botvinick MM, Stenger VA, Carter CS. Anterior cingulate cortex, conflict monitoring, and levels of processing. Neuroimage 2001;14:1302-1308.

34. Dehaene S, Artiges E, Naccache L, Martelli C, Viard A, Schürhoff F, et al. Conscious and subliminal conflicts in normal subjects and patients with schizophrenia: the role of the anterior cingulate. Proc Natl Acad Sci U S A 2003;100:13722-13727.

35. Koechlin E, Summerfield C. An information theoretical approach to prefrontal executive function. Trends Cogn Sci 2007;11:229-235.

36. Chambon V, Franck N, Koechlin E, Fakra E, Ciuperca G, Azorin JM, et al. The architecture of cognitive control in schizophrenia. Brain 2008;131:962-970.

37. Barch D, Ceaser A. Cognition in schizophrenia: core psychological and neural mechanisms. Trends Cogn Sci 2012;16:27-34. 
38. Mann MC, Vaughn AG, Barrantes-Vidal N, Raulin ML, Kwapil TR. The schizotypal ambivalence scale as a marker of schizotypy. J Nerv Ment Dis 2008;196:399-404.

39. Johnson SC, Baxter LC, Wilder LS, Pipe JG, Heiserman JE, Prigatano GP. Neural correlates of self-reflection. Brain 2002;125:1808-1814.

40. Northoff G, Bermpohl F. Cortical midline structures and the self. Trends Cogn Sci 2004;8:102-107.

41. Fellows LK. Orbitofrontal contributions to value-based decision making: evidence from humans with frontal lobe damage. Ann N Y Acad Sci 2011;1239:51-58.

42. Andreasen NC, Paradiso S, O'Leary DS. "Cognitive dysmetria" as an integrative theory of schizophrenia: a dysfunction in cortical-subcortical-cerebellar circuitry? Schizophr Bull 1998;24:203-218.

43. Shinn AK, Baker JT, Lewandowski KE, Öngür D, Cohen BM. Aberrant cerebellar connectivity in motor and association networks in schizophrenia. Front Hum Neurosci 2015;9:134.

44. Der-Avakian A, Markou A. The neurobiology of anhedonia and other reward-related deficits. Trends Neurosci 2012;35:68-77.
45. Hare TA, O’Doherty J, Camerer CF, Schultz W, Rangel A. Dissociating the role of the orbitofrontal cortex and the striatum in the computation of goal values and prediction errors. J Neurosci 2008;28:5623-5630.

46. Gallagher M, McMahan RW, Schoenbaum G. Orbitofrontal cortex and representation of incentive value in associative learning. J Neurosci 1999;19:6610-6614.

47. Strauss GP, Visser KF, Keller WR, Gold JM, Buchanan RW. Anhedonia reflects impairment in making relative value judgments between positive and neutral stimuli in schizophrenia. Schizophr Res 2018;197:156161.

48. Brady RO Jr, Gonsalvez I, Lee I, Öngür D, Seidman LJ, Schmahmann JD, et al. Cerebellar-prefrontal network connectivity and negative symptoms in schizophrenia. Am J Psychiatry 2019;176:512-520.

49. Lui S, Li T, Deng W, Jiang L, Wu Q, Tang H, et al. Short-term effects of antipsychotic treatment on cerebral function in drug-naive first-episode schizophrenia revealed by "resting state" functional magnetic resonance imaging. Arch Gen Psychiatry 2010;67:783-792. 